\title{
New Physical Interpretation of Thermoelectric Cooling in Semiconductor Structures
}

\author{
G. N. Logvinov ${ }^{1}$,Yu. G. Gurevich ${ }^{2 \dagger}$, and José del Rio Valdés ${ }^{1}$ \\ ${ }^{1}$ SEPI-ESIME Culhuacan, Instituto Politécnico Nacional, \\ Av. Santa Ana 1000, C.P. 04430, México, D.F., México, \\ ${ }^{2}$ Dpto. Física Aplicada-Electrônica, Universidad Salamanca, \\ Plaza de la Merced s/n. Edificio Trilingüe, E-37008, Salamanca, Spain
}

Received on 8 December, 2005

\begin{abstract}
Nontraditional approach to explain the thermoelectric cooling is suggested . It is based on the Le ChatelierBraun thermodynamic principle. New effect of cooling and heating of junction of two materials (barrierless thermoelectric cooling) is theoretically predicted, and this effect is different from the Peltier effect (barrier thermoelectric cooling). The suggested thermoelectric effect must be displayed always at the finite values of the junction surface heat conductivity $\eta$. Barrierless thermoelectric effect occurs even in the case when the conducting materials are identical with the same Peltier coefficients. It is shown that both barrier and barrierless thermoelectric cooling effects always exist simultaneously in the general case. The reasons proving reversibility of the thermoelectric cooling process are resulted.
\end{abstract}

Keywords: Thermoelectricity; Thermoelectric cooling

\section{INTRODUCTION}

Traditionally the Peltier effect is explained as absorption of heat or its evolution on junctions of the isothermal structure composed from two different conductors [1] and through which a d.c. electric current flows. This absorption of heat or its evolution occurs in the form of the thermal interaction with external reservoirs providing the isothermality of the structure.

The natural question arises whether the effect Peltier can be displayed in this structure if the areas of the junctions are adiabatically isolated, i.e. in the absence of the thermal interaction with ambient? We are stating that yes, it can be displayed. At that, cooling of the junction or its heating in this case should be understood as decrease of its temperature or increase in comparison with the equilibrium temperature which describes the structure in the absence of an electric current. The opportunity of the temperature increase or decrease in the heterogeneous system through which the electric current flows has the general character and can be understood from the following reasons.

Let us consider the elementary heterogeneous system composed from two different homogeneous semiconductors with $n$-type of conductivity (Fig. 1) through which the electric current $\vec{j}$ runs. We suppose that the electric contacts of the structure $x=-d_{1}$ and $x=-d_{2}$ are kept up at the equilibrium temperature $T_{0}$, the lateral surfaces are insulated adiabatically, and the structure cross-section area is equal to unit everywhere.

The drift heat flux $\vec{q}_{d r}=\Pi \vec{j}$ ( $\Pi$ is the Peltier coefficient) flows together with an electric current through the structure. This flux undergoes discontinuity on the junction of two media unlike to an electric current, and this discontinuity is proportional to $\left(\Pi_{1}-\Pi_{2}\right) \vec{j}$. The drift heat flux either increases or decreases on the junction (depending on a ratio between $\Pi_{1}$ and $\left.\Pi_{2}\right)$ at the given electric current. At that, it is necessary to remember that a sign of the Peltier's coefficients depends on the type of the charge carriers. In semiconductors with $n$-type

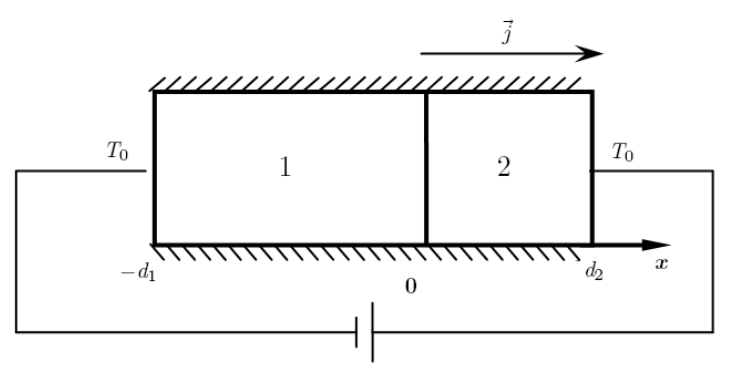

FIG. 1: Semiconductor structure through which a d.c. electric current passes.

of conductivity considering by us, $\Pi<0$.

We claim that according to the general Le Chatelier-Braun principle [2], the other heat fluxes should appear in the structure tending to counteract to these drift fluxes and thereby to reduce this discontinuity. These other thermal fluxes can be only the thermal diffusion fluxes by its nature. Just these fluxes lead to the occurrence of the temperature heterogeneity in the structure. The magnitude of these thermal diffusion fluxes is controlled by the energy balance equation.

The thermal diffusion flux always should coincide in a direction with a heat drift flux in the material of the structure having the less absolute value of $\Pi$. On the contrary, the thermal diffusion flux always should be opposite to a direction to a drift heat flux in a material with the larger absolute value of $\Pi$. Such asymmetry of the directions of the thermal diffusion fluxes in turn leads to its discontinuity on the same junction of the structure. The discontinuity of the thermal diffusion flux in the problem formulated by us (See Fig. 1) necessarily leads to decrease or increase of the temperature on the junction located at $x=0$ (Fig. 2). Just this decrease of the junction temperature or its increase caused by appearance of the induced thermal diffusion fluxes is the essence of the Peltier's effect. For simplification we have assumed here that the surface ther- 


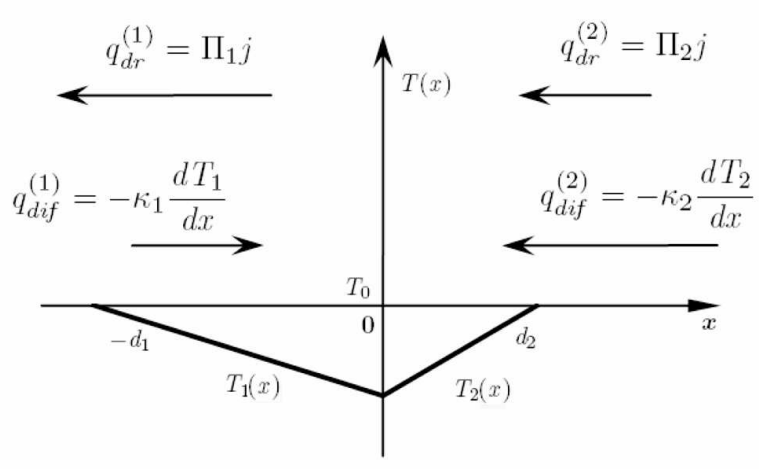

FIG. 2: Thermal fluxes and the temperature distributions in the structure when $\left|\Pi_{2}\right|<\left|\Pi_{1}\right|$.

mal conductivity [3, 4] of the junction is equal to infinity, so the temperature on the junction is continuous.

It is important to notice that the reasoning involved for the explanation of the Peltier effect inevitably lead to occurrence of a new thermoelectric effect of cooling or heating unknown earlier. This effect always takes place at finite magnitude of the surface heat conductivity of the junction and can exist even on the junction of two materials having the same Peltier coefficients. By this reason this effect essentially differs from the Peltier effect and never is reduced to it. We have named this effect the barrierless thermoelectric effect since the mechanism of the cooling in this effect (look below) is not associated with the presence of a build-in-electric field on the junction as it takes place in the Peltier's effect $[5,6]$. This mechanism is determined only by the heat properties of the junction.

To understand the physical essence of the barrierless thermoelectric effect let us consider the structure composed from two identical materials $\left(\Pi_{1}=\Pi_{2}=\Pi\right.$ and $\left.\kappa_{1}=\kappa_{2}=\kappa\right)$. Let us suppose for definiteness that the geometrical sizes of this structure are the same as shown in Fig. 1.

Now the drift heat fluxes are equal in both samples of the structure in magnitude and are directed oppositely to the electric current $(\Pi<0)$.

The induced thermal diffusion fluxes will arise in each sample of the structure tending to counteract to the drift fluxes at finite surface thermal conductivity. It is clear that now the thermal diffusion fluxes will have equal magnitudes and the same direction. The thermal diffusion flux in the left sample always enter into the junction, while the thermal diffusion flux in the right sample always comes out from it. The induced temperature distributions arising in the structure are qualitatively represented in Fig. 3. The temperature gradient is continuous on the interface $x=0$ when $\kappa_{1}=\kappa_{2}=\kappa$ while the temperature is discontinuous here. It is obviously that $\left|T_{1}(x=0)-T_{0}\right| \neq\left|T_{2}(x=0)-T_{0}\right|$ if $d_{1} \neq d_{2}$, so the junction is cooled from one side of the junction and is heated from the other. The final result depends on the sign of the difference $\bar{T}-T_{0}$, where $\bar{T}=\frac{T_{1}(x=0)+T_{2}(x=0)}{2}$ is the average temperature of the junction. There is cooling when $\bar{T}-T_{0}<0$.

The temperatures on both sides of the junction should be

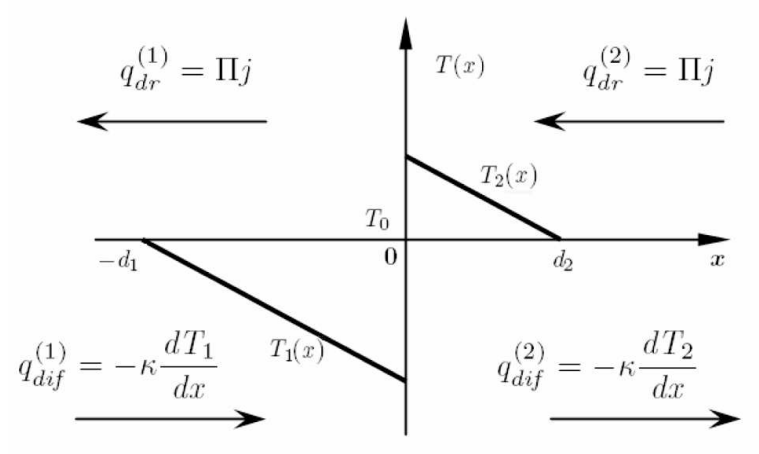

FIG. 3: Thermal diffusion fluxes and the temperature distributions in the barrierless thermoelectric effect $\left(\Pi_{1}=\Pi_{2}, \kappa_{1}=\kappa_{2}\right)$.

continuous if the surface thermal conductivity is infinite. This situation is possible only in the case when $T_{1}(x=0)=T_{2}(x=$ $0)=T_{0}$ (see Fig. 3). It corresponds to the absence of the temperature gradients in both samples of the structure and to disappearance of the barrierless thermoelectric effect. On the contrary, decrease of $\eta$ leads to increase of the temperature break between the temperatures $T_{1}(x=0)$ and $T_{2}(x=0)$.

The basic reasoning regarding the physical interpretation of the barrierless thermal effect remains the same if the structure is made from the different materials. In this case the values of the induced thermal diffusion fluxes on the right-hand side and on the left-hand side of the junction will be different. This circumstance leads now to discontinuity of both the temperatures and the thermal diffusion fluxes on the junction. Moreover the Peltier effect appears in the structure too in this case.

Let us examine the barrierless effect from the quantitative point of view. For simplicity we will restrict ourselves by the structure composed from two identical materials. The basic equation of the problem is the energy balance equation [7] which in the linear approximation in the electric current has the form,

$$
\operatorname{div} \vec{q}=0
$$

where

$$
\vec{q}=-\kappa \nabla T+\Pi \vec{j}
$$

is the generalized thermal flux, and $\kappa$ is the thermal conductivity. Let us emphasize that both coefficients $\kappa$ and $\Pi$ do depend only on the temperature $T_{0}$ in the linear approximation in $\vec{j}$.

In one-dimensional case equation (1) is reduced to

$$
\frac{d^{2} T}{d x^{2}}=0
$$

This equation has to be supplemented by the thermal boundary conditions. As it was mentioned above, for simplicity we have suggested that

$$
T_{1}\left(x=-d_{1}\right)=T_{2}\left(x=d_{2}\right)=T_{0}
$$


The next boundary condition describes the total heat flux conservation at the boundary of two identical materials $q_{1}(x=0)=q_{2}(x=0)$ which is reduced to equation

$$
\left.\frac{d T_{1}}{d x}\right|_{x=0}=\left.\frac{d T_{2}}{d x}\right|_{x=0}
$$

One more boundary condition takes into account the finite magnitude of the surface heat conductivity, $\left.q_{1}\right|_{x=0}=$ $\eta\left[T_{1}(x=0)-T_{2}(x=0)\right][3,4]$ which in accordance with Eq. (2) reduces to the following equation,

$$
\eta\left[T_{1}(x=0)-T_{2}(x=0)\right]=-\left.\kappa \frac{d T}{d x}\right|_{x=0}+\Pi_{j}
$$

The solution of Eq. (3) with boundary conditions [4-6] results in the following temperature distributions,

$$
\begin{gathered}
T_{1,2}(x)=T_{0}\left[1 \pm \frac{\Pi_{j}}{T_{0} \kappa\left(1+\frac{\eta d}{\kappa}\right)}\left(d_{1,2} \pm x\right)\right] \\
\left(-d_{1} \leq x \leq 0 \leq x \leq d_{2}\right)
\end{gathered}
$$

where $d=d_{1}+d_{2}$.

It is easy to see from Eqs. (7) that the temperature $T_{1}$ at the junction $x=0$ is lower than the equilibrium temperature $T_{0}$, while the temperature $T_{2}$ at the same junction exceeds $T_{0}$.

When $\eta \rightarrow \infty$, then, as it was mentioned early, the barrierless effect disappears, so $T_{1}(x)=T_{2}(x)=T_{0}$.

The average temperature on the junction

$$
\bar{T}=T_{0}\left[1-\frac{\Pi_{j}}{2 T_{0} \kappa\left(1+\frac{\eta d}{\kappa}\right)}\left(d_{2}-d_{1}\right)\right]
$$

It is follows from Eq. (8) that heating or cooling of the junction in this case is determined only by ratio of the samples lengths. At equality of these lengths the junction heating from one side is completely compensated by its cooling from the other side.

Let us notice that all equations obtained above are remained correct for the junction of two semiconductors with $p$-type conductivity. It is necessary to mean only that in this case $\Pi_{1,2}>0$.

The barrierless effect exists on the $p-n$ junction too. Let us emphasize that in this case the junction will be cooled down (or heated up) from both sides. However heating or cooling of junction in this case will essential depend on recombination rates in areas adjoined to the junction (see [8]).

Let us notice that both the Peltier effect and the barrierless thermoelectric effect are realized exclusively in the electron subsystem. The electron gas cooling on junction or its heating is caused only by the occurrence of the electron thermal diffusion fluxes and their balance with the drift electron thermal fluxes. The presence of the electron-phonon energy interaction leads to that the heterogeneity of the electron temperature distribution generates the temperature heterogeneity in the phonon subsystem. As a result, the crystal lattice is cooled or heated up too.

The electron and phonon temperatures are equal at infinitely large rates of the electron-phonon energy exchange [9]. In this case the cooling or heating of electrons and phonons occurs as the cooling or heating of a single system. Just this situation has been considered in the present work [see Eqs. $(1-8)]$.

The electron and phonon temperatures are different at the finite rates of the electron-phonon energy interaction [9]. However this fact dose not changes the principle mechanism of the thermoelectric cooling or heating. The difference between the electron and phonon temperatures (two-temperature approximation) leads only to the quantitative change of the effect. The qualitative picture of the cooling remains former.

In summary let us point out the important feature. The discussions about the reversibility of the thermoelectric phenomena are conducted since Thomson [10]. Proportionality of the Peltier's effect to an electric current and, hence, the change of the cooling to heating or vice versa with the change of a direction of an electric current makes natural the assumption that the Peltier effect is the reversible thermodynamic process. On the other hand, it is difficult to consider a process as reversible if it contains an irreversible process of the heat conductivity.

Nevertheless, as it follows from Eq. (1), the entropy flux is conserved in the thermoelectric cooling or heating processes as like as the generalized heat flux when the linear approximation in the electric current is considered and the adiabatic insulation of the structure is maintained on its lateral surfaces,

$$
\operatorname{div} \frac{\vec{q}}{T_{0}}=0
$$

This circumstance unambiguously speaks about the reversibility of the thermoelectric cooling (heating) effect in the linear approximation in the electric current. From our point of view this entropy constancy is associated with the simultaneous presence of two thermal fluxes in the cooling (heating) process, namely with the drift thermal flux and the thermal diffusion flux. The entropy change from the part of one thermal flux is completely compensated by the entropy change from the part of another thermal flux. As a result, the total entropy change of the cooling or heating process is equal to zero. Given statement one can consider as the strict formulation of the Le Chatelier-Braun principle for the thermoelectric processes of cooling or heating.

The process of thermoelectric cooling or heating becomes irreversible if we will take into account the quadratic terms in the electric current in the energy balance equation (the Joule heating and Thomson's effect) or when the "isothermal" Peltier's effect is considered, i.e. when the thermal interaction between the structure and the ambient takes place.

\section{Acknowledgments}

This work has been partially supported by Consejo Nacional de Ciencia y Tecnologia(CONACYT), México 
[1] Neil W. Ashcroft, N. David Mermin, Solid State Physics, Cornell University, Saunders College Publishing, Harcourt Brace Jovanovich College Publishers, Fort Worth Philadelphia San Diego, New York Orlando Austin San Antonio Toronto Montreal London Sydney Tokyo, 1976.

[2] S.R. de Groot and P. Mazur, Non-Equilibrium Thermodynamics (Dover, New York, 1984).

[3] G. D. Mahan and M. Bartkowiak, Appl. Phys. Lett., 74, 953 (1999).

[4] G. N. Logvinov, Yu. G. Gurevich, and I.M. Lashkevich, Jpn. J. Appl. Phys., 42, 4448 (2003).

[5] Jan Tauc, Photo and Thermoelectric Effects in Semiconductors, Pergamon Press, New York-Oxford-London-Paris, 1962.
[6] Yu. G. Gurevich, G. N. Logvinov, O. Yu. Titov, and J. Giraldo, Surface Review and Letters, 9, 1703 (2002).

[7] G. D. Mahan, J. Appl. Phys. 87, 7326 (2000).

[8] I. N. Volovichev, G. N. Logvinov, O. Yu. Titov, and Yu. G. Gurevich, J. Appl. Phys., 95, 4496 (2004).

[9] Yu. G. Gurevich, G. N. Logvinov, A. F. Carballo-Sanchez, Yu. V. Drogobitskiy, and J. L. Salazar, J. Appl. Phys, 91, 183 (2002).

[10] W. Thomson, Mathem. and Phys. Papers, 1, Cambr. Univ. Press (1884).

$\dagger$ On one-year sabbatical leave from CINVESTAV-I.P.N., México D.F. 